Results The majority (15/19) of respondents regarding the competency based training programme attended $\geq 50 \%$ or more of events, compared to only $7 / 24$ in the 2003 curriculum based programme $(p=0.001)$. Of the 11 trainee's in the region, a response rate of $73 \%$ was obtained with a significantly greater proportion (89\%) stating that the competency based training programme met their training needs, compared to $42 \%$ surveyed on the 2003 curriculum based programme $(p=0.001)$. Reasons for lack of attendance included insufficient time to cancel clinical commitments and poor educational value of the 2003 curriculum based programme. Consultant attendance at training meetings increased significantly following redesign of the programme, with $64 \%$ attending $>50 \%$ of meetings compared $12 \%$ prior to instituting a competency based training programme ( $\mathrm{p}=0.004)$. The three most important factors to increase attendance were full day meetings, a consistent venue and keynote speakers from outside of the region.

Conclusion This longitudinal study is the first study to evaluate attendance and educational value of a competency based regional gastroenterology teaching programme. Despite increasing demand to provide service provision and restrictions on study leave allowance, implementation of simple measures as identified in this study may increase consultant attendance and educational quality of regionally organised teaching programmes.

Competing interests None declared.

\section{PTU-266 TRAINEE SATISFACTION WITH JAG E-CERTIFICATION}

doi:10.1136/gutjnl-2012-302514c.266

${ }^{1} \mathrm{~S}$ Dharmasiri, ${ }^{*} \mathrm{C}$ Wells, ${ }^{1} \mathrm{~L}$ Flexer, ${ }^{3} \mathrm{~S}$ Thomas-Gibson, ${ }^{1} \mathrm{P}$ Dunckley. ${ }^{*}{ }^{1}$ Department of Gastroenterology, Gloucester Royal Hospital, Gloucester, UK; ${ }^{2}$ Department of Gastroenterology, University Hospital of North Tees, Stockton on Tees, UK; ${ }^{3}$ Department of Gastroenterology, St Mark's Hospital, London, UK

Introduction In March 2011 JAG launched e-certification allowing trainees to apply for JAG certification through their JETS e-portfolio. Prior to this, trainees were required to submit paper portfolios for application of JAG certification. We report results of a quality assurance survey, which all trainees applying through the JETS eportfolio were invited to complete.

Methods All trainees applying through the JETS e-portfolio were invited to complete an online survey, created to assess trainee experience of e-certification. Trainees were asked if they strongly agreed (SA), agreed (A), were neutral $(\mathrm{N})$, disagreed $(\mathrm{D})$ or strongly disagreed (SD) with statements describing their experience of the process.

Results A total of 109 applications for JAG certification have been made through the JETS e-portfolio since its launch. Some trainees have submitted more than one application for certification in different endoscopic modalities. A total of 33 trainees $(30 \%$ of applications) completed the survey, 7 (21\%) of which had previously applied for JAG certification by the paper or hybrid (paper and eportfolio) methods. When questioned about the general process $69.2 \%$ agreed and $23.1 \%$ strongly agreed that it was better that the previous JAG certification process it replaced. The majority felt that the process was quicker $(\mathrm{SA}=42.9 \%, \mathrm{~A}=42.9 \%)$ and less time consuming ( $\mathrm{SA}=28.6 \%, \mathrm{~A}=57.1 \%$ ). Opinion was divided on cost; $48.5 \%$ agreed that the cost was reasonable and $15.2 \%$ were neutral but $30.3 \%$ disagreed and $6.1 \%$ strongly disagreed. The majority felt that a breakdown of costs would be helpful ( $\mathrm{SA}=18.2 \%, \mathrm{~A}=51.5 \%$, $\mathrm{N}=21.2 \%, \mathrm{D}=9.1 \%$ ). Trainees agreed that the process tested endoscopic competence across all the modalities: gastroscopy $(\mathrm{SA}=37.9 \%, \mathrm{~A}=62.1 \%)$, flexible sigmoidoscopy $(\mathrm{SA}=60 \%, \mathrm{~A}=40 \%)$ and colonoscopy $(\mathrm{SA}=44.4 \%, \mathrm{~A}=55.6 \%)$. There was agreement that the summative assessments were straightforward to submit $(\mathrm{SA}=48.4 \%, \quad \mathrm{~A}=51.6 \%)$ and intuitive $(\mathrm{SA}=32.3 \%, \mathrm{~A}=58.1 \%$,
$\mathrm{N}=9.7 \%$ ). Similarly trainees felt that the process of submitting the application by the JETS e-portfolio was straightforward ( $\mathrm{SA}=42.4 \%$, $\mathrm{A}=54.5 \%, \mathrm{~N}=3 \%$ ), quick ( $\mathrm{SA}=39.4 \%, \mathrm{~A}=54.5 \%, \mathrm{~N}=3 \%, \mathrm{D}=3 \%$ ) and that they were kept informed of their application progress ( $\mathrm{SA}=45.5 \%, \mathrm{~A}=45.5 \%, \mathrm{~N}=9.1 \%$ ).

Conclusion This survey of trainee experience with the JETS e-portfolio application for JAG certification shows that trainees prefer the process compared to the old paper system. It is quicker and trainees agreed the process tested endoscopy competence in gastroscopy, colonoscopy and flexible sigmoidoscopy. The opinion of trainees regarding the cost of the process was spit and trainees would find a breakdown of the cost helpful. The findings of this survey show that the new e-portfolio certification functions well and meets the needs of trainees.

Competing interests None declared.

\section{PTU-267 TRAINING IN GASTROENTEROLOGY IN SEVERN: IMPLEMENTATION OF CHANGE IMPROVES TRAINING}

doi:10.1136/gutjnl-2012-302514c.267

U Chelvaratnam, ${ }^{*}$ E Arthurs, T Mehta, S Pugh, M Lockett. Department of Gastroenterology, Severn Deanery, Bristol, UK

Introduction Feedback on training posts is collected by a variety of different bodies. The Quality Panel was developed to review the quality of gastroenterology training in the region. Feedback on training posts was found to be of variable quality, incomplete, and difficult to analyse. The aim was to create a specific, reproducible method of assessing gastroenterology and general internal medicine (GIM) training, to provide feedback on individual posts and facilitate improvements in training. In 2010 we conducted a survey of training within the region which identified specific areas for improvement which were fed back to individual trusts. This survey was repeated in 2011 to assess for changes.

Methods The 2010 survey comprised of 55 questions within seven domains of gastroenterology and GIM training. Questions were determined based on previous surveys and areas of importance according to consultant and trainee opinion. It was emailed to all registrars in the Severn deanery. Data were collected for the last 3 years of training (2007-2010); preserving anonymity and eliminating bias. Answers correlated to numerical scores, with high scores correlating with high quality. Mean scores were calculated per domain, per trust; a total score was then calculated. The data were presented to the Quality Panel and training committee. Individualised feedback was given to each trust. The survey was repeated in 2011 (with 18 additional questions) to monitor improvements and was analysed with data from 2008 to 2010.

Results The 2010 survey included 37 anonymised responses from 21 trainees at nine hospitals within the Severn region. Responses by trust varied from 3 to 9. Mean overall numerical score was 26.1 (range 24.4-28.6). All trusts scored lowest in providing GIM training with a mean score of 3.1 (range 2.7-3.6); educational support scored highest with a mean score of 4.4 (range $3.4-4.8$ ). The 2011 survey included 46 anonymised responses from 21 trainees at nine hospitals. Responses by trust varied from 3 to 8 . Mean overall numerical score was 26.8 (range 24.7-28.7) and 34.2 (range 31-36.8) with the additional questions. All trusts either improved or retained the same score. All trusts scored lowest in providing GIM training with a mean score of 3.3 (range 2.9-3.8); educational support scored highest with a mean score of 4.5 (range 4-4.8).

Conclusion Creation of a new survey achieved a good response rate and generated speciality specific outcomes and relevant data. This method of assessing training facilitates informed feedback to trusts Repetition of the survey has shown that feedback-led implementation of change has improved training in Severn. By collecting 
data on 3 years of training, improvements may have been underestimated. The methodology is reproducible and may benefit training in other specialities.

Competing interests None declared.

\section{PTU-268 THE IMPACT OF EWTD ON GASTROENTEROLOGY TRAINING AND THE INPATIENT GI REFERRAL SERVICE AT A DISTRICT GENERAL HOSPITAL}

doi:10.1136/gutjnl-2012-302514c.268

V Chhaya, ${ }^{*}$ C Alexakis, M Mendall, S Gupta. Department of Gastroenterology, Croydon University Hospital, London, UK

Introduction A Specialist Registrar's (SpR) weekly duties include two Consultant and one SpR ward round, two clinics, 1-2 endoscopy training lists along with on-calls for General Medicine (GIM). Ward referrals are not included in the timetable. Since the implementation of the European Working Time Directive (EWTD), it has become increasingly difficult to get Gastroenterology (GI) training within the specified working hours. We aim to quantify the impact of EWTD on endoscopy training and the GI referral service at our hospital which serves 350000 people.

Methods Data for GI referrals (excluding GI bleeds and endoscopy requests) was collected by two SpRs from November 2010 to September 2011. All ward referrals were seen within $24 \mathrm{~h}$. An average duration of 20 min was used to see each referral which included a history, examination, review of medical records and documenting a proposed management plan. The average number of weekly referrals and standard rota details were used to analyse the impact of this activity for the year.

Results 515 referrals were seen over a period of 11 months with a mean age of 60 years (range 14-98). 22\% were over 80 years and $54 \%$ were female. On average, 12 referrals were seen per week resulting in a total of $4 \mathrm{~h}$ and $57 \%$ were reviewed by a Consultant. Reasons for referral included liver disease (21\%), inflammatory bowel disease $(15 \%)$, hepatobiliary and pancreatic disease $(13 \%)$, ERCP (13\%), upper GI conditions (12\%), lower GI conditions $(9 \%)$, PEG (8\%) and other miscellaneous conditions (9\%).

Conclusion In a year of 260 working days, 35 days were spent on-call for GIM and 21 for night on-calls (1 in 5 rota). Due to EWTD restrictions, a further 28 days were lost on compensatory leave and 32 on annual leave leaving 144 working days. Only 2.8 days (number of working days remaining/260 working days $x$ number of working days in the week) or 6 sessions per week are spent as a GI trainee. Clinics and ward rounds are fixed commitments leaving trainees to attend endoscopy lists within the remaining time. Due to shift patterns, SpRs are spending more time on the wards. Prospective cover for colleagues produces further challenges. This hinders endoscopy training and leaves referrals to be seen after working hours. We calculate that only $43 \%$ of training lists are attended in the year according to electronic records with endoscopy time recovered on non-training lists. Referrals add $4 \mathrm{~h}$ to the average week and although this is invaluable experience, the time is largely unaccounted for. This survey demonstrates the strains of modern rotas on endoscopy training and ability to see referrals. We suggest appropriate resourcing of the inpatient GI referral service with greater integration between GI and GIM rotas.

Competing interests None declared. 